\title{
IL COORDINAMENTO DELLE PROFESSIONI SANITARIE ALLA LUCE DELLA RECENTE NORMATIVA
}

\author{
Dott Rossetti Filippo ${ }^{1}$
}

${ }^{1}$ Dott. TSRM presso U.O.C. Medicina Nucleare - Azienda Ospedaliera di Rilievo Nazionale e di Alta Specialità "San Giuseppe Moscati", Avellino; Master in Management per le Funzioni di Coordinamento nell'area delle Professioni Sanitarie; Alta Formazione come Incaricato addetto alla Radioprotezione in Ambito Sanitario.

KEYWORDS: Legge 3/2018, comparto sanità, incarichi di organizzazione, incarichi di funzione, CCNL 2016-2018, gestione risorse umane

\section{ABSTRACT}

Il presente lavoro vuole analizzare e trattare gli incarichi di coordinamento alla luce della nuova normativa contrattuale collettiva nazionale 2016-2018- area Comparto Sanità che ridisegna i requisiti e le modalità di svolgimento dei predetti incarichi. Si rende necessario trattare queste argomentazioni che risultano ancora più attuali alla luce della riforma ordinistica della Legge 3/2018 che comprende nei neocostituiti Ordini le Professioni Sanitarie Tecniche, della Riabilitazione e della Prevenzione e che sono, di conseguenza, tematiche trasversali a tutte le 19 Professioni Sanitarie afferenti all'Ordine. Un'altra parte del lavoro tratterà della Gestione delle Risorse Umane, una delle funzioni delle predette figure.

\section{INTRODUZIONE}

Il graduale cambiamento delle organizzazioni sanitarie verso realtà caratterizzate da complessità operative e dalla partecipazione dei diversi operatori ha comportato la necessità di potenziare le funzioni di coordinamento delle risorse umane per razionalizzare le fasi di intervento ed assicurare una risposta con maggiore efficacia. Tali propensioni hanno portato le organizzazioni sanitarie ad approntare modelli funzionali di carattere integrato ed a perfezionare gli strumenti tipicamente aziendali di gestione delle risorse umane, che valorizzino gli aspetti della pianificazione e controllo della produttività, della valutazione delle prestazioni e delle professionalità e ancora dell'analisi dei fabbisogni formativi e del controllo dei costi di budget ecc. Tutto ciò rende evidente che il profilo dirigenziale manageriale, da solo, non è sufficiente a garantire una efficiente ed efficacia funzionalità al sistema di una organizzazione sanitaria se non accompagnato dalla previsione di figure professionali con funzioni di coordinamento che possono contribuire alla gestione aziendale con una visione molto più particolareggiata ed attenta ai bisogni ed alle problematiche. Detto questo, è da considerare che, proprio nell'organizzazione delle aziende sanitarie, i dirigenti di vario livello non sono gli unici leader dei lavoratori, ma maggior ragione lo possono essere anche i coordinatori di livello intermedio con i quali i dipendenti entrano maggiormente e quotidianamente in contatto e verso i quali si richiedono non solo capacità di tipo tecnico-assistenziale, ma anche doti di comunicazione, motivazione e capacità di far partecipare gli operatori ai processi decisionali. Con il presente lavoro si vuole analizzare la funzione di coordinamento del personale tecnico-sanitario, prendendo in considerazione dapprima la specifica natura oltre che le caratteristiche contrattuali ed, in seguito, le specifiche competenze organizzative e gestionali.

\section{METODOLOGIA E MATERIALI}

Nel campo normativo particolare evidenza assume la Legge 1 Febbraio 2006, n. 43 "Disposizioni in materia di professioni sanitarie infermieristiche, ostetriche, riabilitative, tecnico-sanitarie e della prevenzione e delega al Governo per l'istituzione dei relativi ordini professionali", la quale all'articolo 6, comma 1 lettera b) individua l'area dei professionisti coordinatori in possesso del master di primo livello in management o per le funzioni di coordinamento rilasciato dall'università (ai sensi dell'articolo 3, comma 8, del regolamento di cui al decreto del Ministero dell'università e della ricerca scientifica e tecnologica 3 Novembre 1999, n. 509, e dell'articolo 3, comma 9, del regolamento di cui al decreto del Ministro dell'istruzione, dell'università e della ricerca 22 Ottobre 2004, n. 270). La recente normativa valorizza appositamente la natura manageriale dei professionisti coordinatori, identificandoli come apposita articolazione professionale che necessita di una formazione specifica di management, distinta dalla formazione dei professionisti di base e dei professionisti specialisti. Le funzioni di coordinamento del personale sono state previste, prima dell'attuazione del recente Contratto Collettivo Nazionale di Lavoro 2018, dall' art. 10 del Contratto Collettivo Nazionale di Lavoro, II biennio economico 2001-2002, siglato il 20.09.2001 - area comparto sanità, che dispone una specifica indennità con assunzione di responsabilità del proprio operato. La natura delle funzioni di coordinamento si inquadra in una funzione direttiva ed organizzativa che ha come caratteristica principale e saliente quella di realizzare la concreta sinergia ed integrazione dei vari interventi necessari al corretto funzionamento delle attività dei servizi di assegnazione. In tale contesto, l'Azienda Sanitaria, attuando un'analisi organizzativa delle diverse funzioni di coordinamento da attivare, deve effettuare una selezione in base a:

- il grado di livello del coordinamento da effettuare: intendendo con ciò se il coordinamento riguarda l'intera articolazione aziendale oppure uno specifico dipartimento aziendale o, ancora, uno specifico servizio o una unità operativa semplice;

- il numero delle persone da coordinare e gli specifici contesti di intervento, stabilendo, quindi, la 
complessità del coordinamento del personale in base ad una dimensione puramente quantitativa o anche legata a specifici interventi caratterizzati da una certa complessità funzionale;

- la complessità della tecnologia utilizzata;

- la complessità prestazionale;

- la tipologia dell'utenza;

- la presenza o meno di relazioni complesse.

Effettuata tale analisi, si giunge successivamente alla fase saliente di scelta delle persone che meglio rispondono alle caratteristiche della funzione. Si possono effettuare, quindi, nel principio di trasparenza ed imparzialità, delle selezioni interne tra il personale interessato a ricoprire la funzione, prevedendo delle regole chiare, semplici e soprattutto efficaci nella scelta delle persone ritenute più idonee allo svolgimento della delicata funzione di coordinamento. In tale ambito, è da precisare quanto previsto dall'articolo 4 della Legge n. 43/2006 citata, il quale prevede, per le future e nuove funzioni di coordinamento da assegnare, il possesso dei seguenti requisiti: a) master di primo livello in management o per le funzioni di coordinamento nell'area di appartenenza; b) esperienza almeno triennale nel profilo di appartenenza. Anche il coordinatore è coinvolto in maniera diretta nel processo funzionale aziendale di monitoraggio delle risorse economiche che si hanno a disposizione attraverso i documenti di budget. In tale ambito, il budget della struttura operativa di riferimento, nel quale sicuramente il primo livello di responsabilità compete al dirigente responsabile della struttura, non può non tenere conto dell'importante apporto funzionale, operativo e di trasmissione dei dati che compete sicuramente al coordinatore per il proprio ambito di attività. Pertanto, valorizzare l'aspetto economico e promuovere dei metodi comportamentali atti a ridurre gli sprechi e le inefficienze è per il coordinatore un compito essenziale che produce effetti significativi nel risultato complessivo di tutta l'unità operativa.

Altro aspetto importante risulta essere quello relativo alla garanzia ed alla sicurezza negli ambienti di lavoro, che per poter essere efficace deve prevedere la collaborazione delle risorse umane materialmente più vicine agli ambiti produttivi. Tale presupposto richiede il necessario coinvolgimento del personale con funzioni di coordinamento in una accurata introduzione di metodi comportamentali nei confronti di ogni operatore atti a segnalare eventuali disfunzioni tecniche delle apparecchiature o eventuali deficit strutturali che possono inficiare la sicurezza nei luoghi di lavoro o ancora esigere il rispetto da parte di tutti i componenti dell'equipe di riferimento dei dispositivi minimi di sicurezza.

Per quanto riguarda il Contratto Collettivo Nazionale di Lavoro del comparto Sanità 2018, siglato negli ultimi mesi, le carriere gestionali, trattate dall'art.14 all'art.23, vengono ridisegnate, mentre per la prima volta, si tenta di istituire, meritoriamente, le carriere clinico-professionali. Tutto il sistema abbandona definitivamente la classica impostazione caratterizzata dalla carriera verticale a favore del sistema privatistico degli incarichi di funzione attribuibili con selezione interna, sottoposti a valutazione e revocabili. Da quanto si può desumere dal CCNL 2018, artt. 14-23, vi saranno due tipologie di carriere: una, più consolidata nel tempo, di carattere organizzativo e l'altra, che si presenta come la vera novità, di carattere professionale. Gli incarichi di funzione si dividono in "incarichi di organizzazione" e in "incarichi professionali". Il principio di carattere generale relativo agli incarichi viene determinato dalla stessa normativa contrattuale che recita testualmente: "gli incarichi richiedono anche lo svolgimento di funzioni con assunzione diretta di elevata responsabilità aggiuntive e/o maggiormente complesse rispetto alle attribuzioni proprie della categoria e del profilo di appartenenza". Si antepone una maggiore responsabilità alle attribuzioni. Gli incarichi di funzione, di organizzazione e professionali vengono attribuiti in base a procedure selettive aziendali e sono conferiti con "atto scritto e motivato" che ne riporta i contenuti con la "descrizione delle linee di attività". L'incarico è "a termine" con una durata che va da tre a cinque anni. È revocabile, prima del termine per tre motivi:

a) soppressione del posto per modifica dell'atto aziendale;

b) valutazione negativa (evidentemente intermedia);

c) per mancanza sopraggiunta dei requisiti di attribuzione.

Le indennità rimangono ferme al dato storico degli incarichi organizzativi del 1999 e del 2001, la valutazione è prevista annualmente, come valutazione intermedia, e al termine dell'incarico. La valutazione negativa viene riportata nel fascicolo personale e costituirà un precedente per l'affidamento di altri incarichi. Prima della formalizzazione negativa, deve essere previsto un "contraddittorio" con il lavoratore. Il testo contrattuale parla della valutazione positiva come presupposto di "altri incarichi", non chiarendo se possa anche essere rinnovato lo stesso incarico. Rispetto all'assetto attuale vengono ridefiniti gli ex incarichi di coordinamento e di posizione organizzativa creando una complessiva specifica normativa sugli "incarichi di organizzazione" all'interno del più generale istituto sugli "incarichi di funzione". Si può leggere testualmente dal CCNL: "l'incarico di organizzazione comporta l'assunzione di decisioni necessarie per affrontare problemi complessi nel governo dei processi assistenziali e formativi connessi all'esercizio della funzione sanitaria e sociosanitaria" e viene distinto in "meno complesso" relativo alla funzione di coordinamento e in "più complesso" relativo alla posizione organizzativa. Cambiano i requisiti solo per l'incarico di posizione organizzativa. E', infatti, richiesto il requisito "della laurea specialistica o magistrale e cinque anni di esperienza professionale nel profilo di appartenenza e in categoria D”. Nulla cambia invece per il coordinamento in cui si richiede il relativo master previsto dalla normativa vigente e tengono conto di una serie di aspetti quali la dimensione organizzativa, il livello di autonomia richiesto e altre caratteristiche. Infine, per entrambi gli incarichi di funzione, di coordinamento e di posizione organizzativa, l'indennità "assorbe il compenso per il lavoro straordinario". Per gli incarichi "più complessi" relativi alle posizioni organizzative la novità più rilevante è relativa al requisito della laurea magistrale. Gli incarichi professionali, invece, sono la vera novità del sistema di incarichi complessivo ridisegnato dal CCNL 2016-2018, tant'è che già nel contratto del 1999 le parti si posero il problema inventando la "carriera orizzontale", c.d. a fasce, che avrebbero dovuto, nelle intenzioni, essere una sorta di riconoscimento di carriera clinico-assistenziale. L'incarico professionale può essere di "professionista specialista" o di "professionista esperto". Si può leggere testualmente che "nell'ambito 
delle specifiche aree di intervento delle professioni sanitarie infermieristiche, tecniche, della riabilitazione, della prevenzione nonché della professione di ostetrica $e$ in relazione alle istituende aree di formazione complementare post diploma, sono istituiti incarichi professionali per l'esercizio di compiti derivanti dalla specifica organizzazione delle funzioni delle predette aree prevista nell'organizzazione aziendale. Tali compiti sono aggiuntivi elo maggiormente complessi $e$ richiedono significative, elevate ed innovative competenze professionali rispetto a quelle del profilo posseduto". Requisito per il professionista specialista è il master di primo livello, mentre requisito per il conferimento dell'incarico di "professionista esperto" è costituito dall'aver acquisito, "competenze avanzate, tramite percorsi formativi complementari regionali ed attraverso l'esercizio di attività professionali, anche in virtù di protocolli concordati tra le regioni e le rappresentanze istituzionali delle professioni mediche, sanitarie e sociosanitarie interessate". I livelli di riconoscimento sono quindi due: a) le competenze specialistiche, a cui non serve alcun atto successivo e di attuazione in quanto, avendo individuato il requisito di accesso, il master specialistico, se ne intende individuato anche il campo di attività. Non vi sono dubbi che tale soluzione sia sostanzialmente in linea con il dettato legislativo e, in particolare, con la legge 42/99 che nella formazione post-base individua uno degli elementi per l'ampliamento del "campo proprio di attività e responsabilità"; b) le competenze avanzate sono, invece, più complesse da individuare e sono il presupposto per l'incarico di professionista esperto. Qui, la formazione post-base è di carattere regionale e si accompagna "attraverso l'esercizio di attività professionali", da intendersi, evidentemente, come aggiuntive.

\section{Risultati e discussione}

Le caratteristiche delle funzioni di coordinamento costituiscono una occasione importante per le aziende sanitarie per approntare nuovi modelli organizzativi rendendo, in questo modo, concreta la realizzazione diuna vera dirigenza intermedia, anello di congiunzione fra gli obiettivi aziendali e gli operatori. L'organizzazione delle attività costituisce una delle prime importanti funzioni dei coordinatori delle aziende sanitarie. Ciò inizia con la scelta di un modello cui fare riferimento nella scelta della funzione organizzativa che sia la più appropriata all'ambiente di riferimento, alla tipologia di utenza ed alle tipologie delle prestazioni. Tra i modelli più evoluti esiste quello denominato case management consistente in un processo di erogazione di prestazioni sanitarie incentrato sul cliente e avente l'obiettivo di integrare con la massima efficacia gli interventi necessari ai fini di garantire un'assistenza appropriata che migliori la qualità della vita ed il contenimento dei costi. Una volta scelto il modello organizzativo più appropriato, si passa alla fase di pianificazione delle attività. Pianificare significa scegliere preventivamente gli obiettivi ed $\mathrm{i}$ relativi percorsi, che un sistema organizzativo deve realizzare in un dato periodo di tempo. Tale funzione di pianificazione avviene in modo scritto o attraverso l'utilizzo di strumenti telematici, in modo formale e portato a conoscenza di tutti i componenti dell'equipe di riferimento. Nell'ottica della pianificazione delle attività, si tende ad indurre i collaboratori di guardare al di là dei bisogni immediati e delle emergenze, individuando i risultati attesi e i modi per ottenerli.
Anzi, proprio una buona pianificazione riduce lo stress e le tensioni provocate dalle situazioni di crisi e dalle emergenze, in quanto prepara il personale anzitempo su quali strumenti adottare e come comportarsi. I leader, pertanto, inducono le persone a voler fare piuttosto che a voler essere. Il punto qualificante del leader tende verso l'attenzione al miglioramento della perfomance dei collaboratori ed al pieno sviluppo di tutto il potenziale cui essi sono portatori, riuscendo a motivare le persone a fare di più di quanto esse avessero intenzione di fare e spesso di più di quanto esse ritenessero possibile fare, facendo leva sui bisogni più elevati delle persone quali la stima e l'autorealizzazione. I fattori funzionali essenziali relativi all'organizzazione, gestione e valutazione delle risorse umane, da parte dei coordinatori, sono i seguenti: a) analisi di fabbisogno delle risorse umane necessarie: in tale contesto la funzione di coordinamento, essendo quella di più immediato contatto con l'equipe operativa, deve valutare, analizzare ed infine segnalare ai livelli di responsabilità dirigenziale superiore fino al servizio infermieristico e tecnico aziendale ed al servizio risorse umane, la necessità di reclutamento di nuove risorse, collaborando attivamente al fine di raggiungere l'obiettivo principe del reclutamento che è quello di riuscire ad inserire la persona giusta al posto giusto. Tale funzione richiede anche un'attenzione particolare una volta che la risorsa umana è stata acquisita, al fine di organizzare un efficace inserimento con l'accostamento di un tutor che accompagnerà la nuova unità nell'ambiente di riferimento. Conoscere le persone, conoscere le esigenze funzionali assistenziali specifiche di una determinata unità operativa è un compito essenziale del coordinatore che risulta in tal modo essere la figura informativa essenziale di congiunzione con la restante dirigenza aziendale, al fine di intraprendere azioni mirate ed efficaci.

b) analisi delle posizioni operative ed organizzazione funzionale: tale funzione richiede, in correlazione con il modello funzionale-assistenziale scelto e all'ambiente organizzativo di riferimento della unità operativa, la costruzione di una sorta di organigramma funzionale ove sono indicate le posizioni operative. In aggiunta, è essenziale accostare all'organigramma i vari protocolli operativi (linee guida, regolamenti ecc.) che descrivono l'insieme di regole operative.

c) elaborazione dei piani di lavoro: il piano di lavoro è lo strumento diretto alla programmazione a breve termine dell'unità operativa di riferimento. Attraverso il piano di lavoro il coordinatore deve dapprima definire l'obiettivo generale, avente una finalità ampia e congruente con gli obiettivi previsti dalla programmazione strategica aziendale. Di conseguenza si procederà alla definizione di obiettivi specifici e di risultato e delle relative azioni da svolgere per la loro realizzazione. L'accostamento delle risorse umane con il piano di lavoro risulta, poi, essenziale nella precisa individuazione del numero quantitativo e delle professionalità da dedicare alle specifiche azioni previste. In ciò può essere determinante, proprio per il fattore motivazionale, coinvolgere direttamente le risorse umane al momento dell'affidamento dei compiti e delle relative azioni previste nei piani di lavoro, ascoltando il loro parere e soprattutto le loro proposte di miglioramento e superamento di eventuali criticità. In ciò si rende un sistema funzionale basato sulla vera dinamicità e attività delle risorse umane, diretto a valorizzare l'apporto di ognuno nelle analisi di feedback e nella immediata segnalazione di problematiche 
dell'attività ordinaria di esecuzione dei piani di lavoro. d) valutazione della perfomance professionale: la valutazione in tale ambito attiene alla specifica attività tecnico-assistenziale del personale di riferimento. In tal caso possono essere presi a riferimento dei criteri di valutazione quali: la prestazione e la precisione, l'aspetto relazionale e di lavorare per gruppi, il rispetto dei criteri e protocolli operativi, il lavoro multiprofessionale ed integrato, il grado di umanizzazione e accoglienza degli utenti, il grado di collaborazione con i superiori ecc.

e) valutazione della produttività collettiva: tale aspetto concerne la capacità o meno di raggiungere determinati obiettivi, prefissati di solito all'inizio dell'esercizio di riferimento cui sono legate specifiche risorse economiche (sistema premiante). Il processo di valutazione della produttività, che va distinto da quello della prestazione professionale ordinaria di cui sopra, deve necessariamente prevedere una pianificazione degli obiettivi chiari e misurabili, condivisi dal personale di riferimento o quantomeno comunicati prima del loro inizio. Successivamente, è necessario prestare particolare attenzione alla fase di monitoraggio, ove è essenziale che si attuino dei metodi comportamentali tendenti a segnalare immediatamente eventuali ipotesi di scostamento degli obiettivi per poi approntare in tempo eventuali elementi correttivi. Infine, il processo deve prevedere la fase importante di valutazione dei risultati, con la discussione tra gli operatori dei successi ottenuti o anche dei risultati non ottenuti, analizzando, con ciò, le cause degli scostamenti e approntando con questo gli elementi necessari per far sì che, al prossimo esercizio, il risultato sia migliore. Infine bisogna evidenziare che al raggiungimento dell'obiettivo non è sufficiente erogare il relativo premio economico, ma un'azione efficace richiede la capacità di erogare $\mathrm{i}$ cosiddetti incentivi morali (che spesso danno più soddisfazione alle risorse umane), comunicando il proprio soddisfacimento per l'importante obiettivo raggiunto e rendendo evidente quanto quella risorsa umana o quel gruppo è risultato determinante per il risultato.

f) valutazione del potenziale professionale: conoscere le proprie risorse umane, sapere i punti di forza e di debolezza contribuisce a tarare meglio l'organizzazione e costruire il possibile trend prestazionale. Tale analisi è sicuramente un punto di partenza necessario del coordinatore sulla costruzione di percorsi di sviluppo del potenziale professionale, attraverso l'analisi dei fabbisogni formativi e la collaborazione nei processi di sviluppo delle carriere;

g) analisi dei fabbisogni formativi: una delle funzioni di preminente importanza del coordinatore attiene alla collaborazione nell'elaborazione del programma di formazione aziendale. La funzione di coordinamento deve essere coinvolta in prima battuta da parte del dirigente responsabile dell'unità operativa di riferimento per l'elaborazione del fabbisogno formativo delle unità di risorse umane. In ciò è importante collegare, in un processo integrato, la funzione della formazione con quella della valutazione del personale, in modo da visualizzare i punti di forza e di debolezza dell'operatore e giungere così a definire una sorta di formazione mirata a colmare proprio i punti di debolezza.

h) analisi di benessere organizzativo: la funzione di coordinamento, essendo quella di più immediato contatto con l'ambiente lavorativo di riferimento, è un punto essenziale nell'analisi informativa sulle condizioni ambientali di lavoro, sui rapporti inter-personali e in generale sulle criticità esistenti nell'organizzazione, al fine di avanzare proposte di miglioramento che tendano al benessere organizzativo.

Tali funzioni debbono prevedere una sorta di manuale della qualità accettato e condiviso come strumento importante per la diffusione di una cultura secondo la quale la qualità è un elemento essenziale dell'attività professionale. Oggi nelle aziende sanitarie si assiste a dei percorsi assistenziali caratterizzate da una forte integrazione degli interventi (sia a livello interno che esterno, dipartimenti ospedalieri, dipartimenti di cure primarie territoriali, servizi socio-assistenziali, servizi ospedalieri/servizi territoriali) fondati spesso su approcci multiprofessionali nella risposta ai problemi di salute. Da ciò il vantaggio competitivo di ogni Azienda Sanitaria si fonderà anche sulla capacità di integrazione delle diverse componenti professionali, favorendo e valorizzando al massimo le funzioni di coordinamento che a volte costituiscono proprio il primo livello di integrazione nei punti di immediata erogazione delle prestazioni ai cittadini. Nella maggior parte delle Aziende Sanitarie, però, non è ancora matura la convinzione che, per durare nel tempo e svilupparsi, è essenziale porre più attenzione alle particolari capacità dei ruoli intermedi e alle loro abilità di attivare efficaci funzioni di coordinamento del personale. Ciò che manca ancora, in alcune realtà, è una precisa politica aziendale verso lo sviluppo del ruolo dei quadri intermedi sanitari al fine di raggiungere maggiori livelli di funzionalità di servizio. Una sfida difficile e importante che richiede un notevole investimento da parte delle Aziende Sanitarie al fine di evitare di costruire figure formali di coordinamento, realizzando, gradualmente nel tempo, reali funzioni di coordinamento che saranno determinanti per un miglioramento organizzativo e, con ciò, qualitativo delle prestazioni assistenziali ai cittadini.

\section{REFERENCES}

1. AA.VV. Cerismas, (2010). "Lo sviluppo delle pratiche di Human Resource Management in Sanità"; Workshop 17 Dicembre 2010;

2. ASS n.6 Friuli Occidentale, (2007). "Il profilo delle competenze del Coordinatore";

3. CCNL - area Comparto Sanità 2001-2002 e 2016- 2018;

4. Cerismas, (2011). "Ruoli e competenze nella sanità: lo sviluppo delle pratiche di Human Resource Management: scenari ed esperienze di successo", Workshop 21 giugno 2011;

5. Conte Anna, Fiorani Gloria - Rendere conto ai cittadini. La gestione delle risorse umane in sanità, "Infermieristica e Lean Thinking. Metodologie e strumenti per una corretta assistenza", AA.VV., (2017). "La gestione delle risorse umane in sanità: il modello di gestione per competenze", 7. Rebora G. (2004), "I capi azienda, modelli emergenti di management". Milano, Guerini Associati;

6. Dossier Regione Emilia-Romagna, (2014). "Risorse Umane in Sanità" - DOSSIER 239-2014 ISSN 1591-223X, "Sviluppo delle professionalità per l'assistenza e la salute", 2014;

7. Tanese A., (2002). “L'evoluzione delle funzioni innovative nelle aziende del SSN: riflessioni conclusive”, Mecosan 11 (43): $117-126$. 